\title{
Top 5 Trends in DSM for the Next Decade
}

Jean-François Masse, DMD, MSc, FACD, Diplomate, ABDSM

Editor-in-Chief Journal of Dental Sleep Medicine

Universite Laval, Quebec City, Quebec, Canada

As the new year begins, I believe we can now take a few moments to think about what sleep dentists should expect entering a new decade. Nobody truly knows what the future will hold and attempting to predict it is always hazardous. For that reason, I have asked a few experts in the field for their opinion. The suggestions that they gave me are very interesting and I just had to, as the late Steve Jobs advised in his famous speech to Stanford's graduates, "connect the dots." I I wish dental sleep medicine was that simple...here we go. Do not blame me if I am proven wrong:

\section{Dental Sleep Medicine, direct to consumer style}

I have nothing to say about specific direct to consumer companies, neither good nor bad. It is a business model. When you look at the number of people suffering from sleep apnea, ${ }^{2,3}$ it is just a matter of time before a similar direct-to-consumer company steps in.

What can be done? It is hard to say, but a few things come to mind. Continue educating ourselves. Get in touch with the labs who are our partners to improve common expertise. Demonstrate to our medical specialists the value of our work. Support and endorse the membership of the AADSM. It is critical for us to unite and the time is now.

\section{Major changes in the way sleep medicine cases are processed}

The number of OSA patients is overwhelming, yet the number of medical specialists equipped to diagnose and treat them is far from adequate. ${ }^{4}$ This needs to be urgently addressed by our medical colleagues. Sleep apnea has become a visible public health problem and with the prevalence of obesity on the rise among other factors, it will only get worse.

The rationale alone for treating OSA patients has changed significantly in the last few years. ${ }^{5,6}$ Isn't it time to also review the way patients are handled? I strongly invite my colleagues to read the letter to the editor in this edition of the $\mathrm{JDSM}^{7}$ and give your comments.

\section{Lowering of the cost of oral appliance therapy for the patient}

Costs will decrease when a better case selection is performed by sleep dentists. This improved case selection will allow us to spend less chair time on unsuccessful patients, and, paradoxically, less time on successful cases as well.

Phenotyping, incidentally, had been previously presented as a promising yet time consuming, techniquesensitive, costly procedure. A recent study has shown that PSG signals can now be used for phenotyping in a retrospective and relatively inexpensive way $^{8}$, thus making the technique more accessible both clinically and financially.

Better case selection, in addition, will promote interdisciplinary approaches, once again reducing overall cost. The use of trial appliances, techniques like the Matrx or $\operatorname{DISE}^{9}$ along with phenotyping ${ }^{10}$ must be explored by the sleep dentist.

4. Technology: telemedicine, oral 3D scanning, 3D printing, compliance monitors, sleep apps and artificial intelligence

Technology is everywhere. This category should be an editorial in and of itself. The efficiency and accessibility of these tools is increasing while their cost continues to diminish. It is hard to predict where technology will progress, but it is certain that its impact will be tremendous in ways we cannot imagine as of yet.

\section{Research, more research}

Not so long ago, we had to educate the public on a daily basis regarding obstructive sleep apnea, CPAPs, and what is the dentist's role in treating OSA. This is no longer the case, as public awareness has increased exponentially, now with an understanding of the potential impact of sleep apnea on their lives.

There is still, however, obviously a lot to be learned. It seems that the more we know about the subject, the more questions arise. Expect more resources to be directed towards the study of sleep disorders, especially obstructive sleep apnea. 
To all my colleagues, I wish you a happy new year and look forward to discussing with you by email or in person about the subjects you feel are important for our community.

Communication is the key,

JF Masse, DMD, MSc, FACD, D.ABDSM

\section{CITATION}

Masse, JF. Top 5 trends in DSM for the next decade. $J$ Dent Sleep Med. 2020;7(1)

\section{REFERENCES}

1. 'You've got to find what you love', Jobs says. Stanford News. https://news.stanford.edu/2005/06/14/jobs-061505/. June 14, 2005. Accessed January 7, 2020.

2. Masse, JF. With 'epidemic' rise in OSA, should qualified dentists provide CPAP therapy? J Dent Sleep Med. 2019;6(4)

3. Benjafield A V, Ayas NT, Eastwood PR, et al. Estimation of the global prevalence and burden of obstructive sleep apnoea: a literature-based analysis. Lancet Respir Med. 2019;7(8):687-698. doi:10.1016/S2213-2600(19)30198-5

4. American Board of Medical Specialties. ABMS Board Certification Report : 2018-2019. 2019. https://www.abms.org/media/257753/abms-board-certificationreport-2018-2019.pdf. Accessed January 7, 2020.

5. McEvoy RD, Antic NA, Heeley E, et al. CPAP for prevention of cardiovascular events in obstructive sleep apnea. $N$ Engl J Med. 2016;375(10):919-931.

6. Yu J, Zhou Z, McEvoy RD, et al. Association of positive airway pressure with cardiovascular events and death in adults with sleep apnea: a systematic review and meta-analysis. JAMA.

2017;318(2):156-166

7. Simmons MS, Shapiro CM. The New DDS - "Dentists Diagnosing Sleep." J Dent Sleep Med. 2020;7(1)
8. Won CH, Reid M, Sofer T, et al. Sex Differences in Obstructive Sleep Apnea Phenotypes, the Multi-Ethnic Study of Atherosclerosis. Sleep. 2019. doi:10.1093/sleep/zsz274

9. Dieltjens M, Braem MJ, Op De Beeck S, et al. Remotely controlled mandibular positioning of oral appliance therapy during polysomnography and drug-induced sleep endoscopy compared with conventional subjective titration in patients with obstructive sleep apnea: Protocol for a randomized crossover trial. Trials. 2019;20(1). doi:10.1186/s13063-019-3698-4

10. Lai V, Carberry JC, Eckert DJ. Sleep Apnea Phenotyping: Implications for Dental Sleep Medicine. J Dent Sleep Med. 2019;6(2)

\section{SUBMISSION AND CORRESPONDENCE INFORMATION}

\section{Submitted in final revised form January 6, 2020.}

Address correspondence to: Jean-François Masse, DDS, MSc, FACD, D.ABDSM, Professor, Universite Laval, 2780 Masson \#200, Quebec City, QC, G1P 1J6, Canada; Tel: 418871-1447; Fax: 418-871-4983; Email: jean-francois.masse@fmd.ulaval.ca 